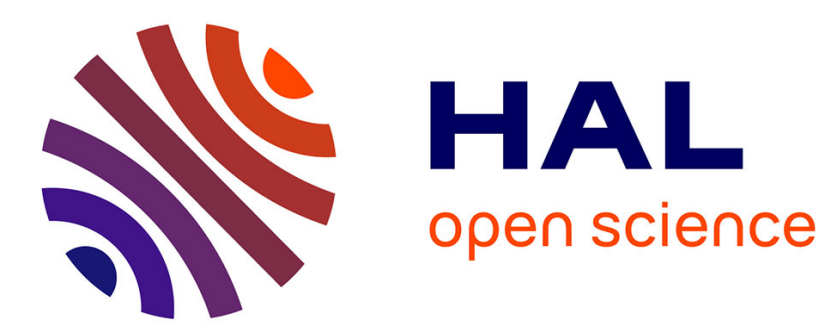

\title{
Influence of Centrifugal Forces on Oil Flow in Journal Bearing of Planetary Gear
}

\author{
Mikhail Temis, Alexander Lazarev
}

\section{To cite this version:}

Mikhail Temis, Alexander Lazarev. Influence of Centrifugal Forces on Oil Flow in Journal Bearing of Planetary Gear. 16th International Symposium on Transport Phenomena and Dynamics of Rotating Machinery, Apr 2016, Honolulu, United States. hal-01894395

\section{HAL Id: hal-01894395 https://hal.science/hal-01894395}

Submitted on 12 Oct 2018

HAL is a multi-disciplinary open access archive for the deposit and dissemination of scientific research documents, whether they are published or not. The documents may come from teaching and research institutions in France or abroad, or from public or private research centers.
L'archive ouverte pluridisciplinaire HAL, est destinée au dépôt et à la diffusion de documents scientifiques de niveau recherche, publiés ou non, émanant des établissements d'enseignement et de recherche français ou étrangers, des laboratoires publics ou privés. 


\title{
Influence of Centrifugal Forces on Oil Flow in Journal Bearing of Planetary Gear
}

\author{
Mikhail Temis $^{1,2 *}$, Alexander Lazarev ${ }^{1,2}$
}

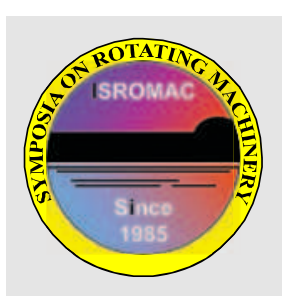

ISROMAC 2016

International

Symposium on Transport

Phenomena and Dynamics of

Rotating Machinery

Hawaii, Honolulu

April 10-15, 2016

\begin{abstract}
Mathematical model of oil flow in fluid film bearing in field of centrifugal forces is developed. Centrifugal forces for planet wheel bearing sliding surfaces and oil gap are formulated. This model is based on modification of two-dimensional Reynolds equation taking into account inertia centrifugal forces for oil film. Required modification of Reynolds equation is received from Navier-Stokes and continuity equations taking into account centrifugal forces acting on planet wheel bearing. Modified 2D Reynolds equation is solved numerically using finite-element discretization.
\end{abstract}

Developed mathematical model is verificated at comparison with solution of full Navier-Stokes equations system obtained in commercial software package. Results for pressure distribution for bearing with fixed axis and for planet wheel bearing are received and compared. The sufficient influence of centrifugal inertia forces in oil layer of planet wheel bearing on pressure distribution, bearing carrying force and attitude angle is shown for specific shaft journal eccentricity ratio, eccentricity direction and rotation velocity.

\section{Keywords}

Fluid film bearing - Centrifugal inertia forces - Reynolds equation

\footnotetext{
${ }^{1}$ Central Institute of Aviation Motors, Moscow, Russia

${ }^{2}$ Bauman Moscow State Technological University, Moscow, Russia

*Corresponding author: mikhail.temis@gmail.com
}

\section{INTRODUCTION}

Planetary gears have a wide application in different areas of industry. At present moment, they find application in perspective geared turbofan aircraft engine [1]. Integration of planetary gear in gas turbine engine as a mechanism for transmitting of rotation from low-pressure rotor to fan rotor gives an opportunity for choosing of optimal aerodynamic and structural parameters of both rotors [2]. In present moment a single-row planetary gear (Figure 1a) is used for current purposes. Fluid film bearings used in supports for high speed or heavy-loaded gears. Main characteristics of fluid film bearing such as stiffness and damping based upon hydrodynamic fluid pressure caused by relative motion of sliding surfaces. In most of applications, fluid inertia forces are negligible in comparison with hydrodynamic forces, but if some applications their influence on bearing characteristics could be sufficient and should be taken into account. Planet wheel journal bearing are an example where influence of fluid inertia forces have an influence on bearing characteristics.

Parameters of fluid flow in bearing with fixed axis usually calculated by Reynolds equation [3]. However, conventional Reynolds equation form received from NavierStokes and continuity equations while neglecting inertia forces. Sufficient linear velocity of planet wheel bearing rotating around carrier velocity leads to increasing of contribution of centrifugal inertia forces in pressure distribution in bearing, bearing carrying force value and direction. For taking into account inertia forces acting on oil film in journal bearing of planet wheel the conventional mathematical model for oil flow in journal bearing based on Reynolds equation requires modification.

\section{INERTIA FORCES}

For any type of planetary gear each planet wheel (2) participating in compound motion: rotation with carrier $(H)$ with angular speed $\omega_{H}$ and rotation about self-axis with angular speed $\omega_{2}$ (Figure 1a). Relation between carrier and planet wheel angular speeds for single-row planetary gear is as follows

$$
\frac{\omega_{2}}{\omega_{H}}=1-\frac{z_{3}}{z_{2}},
$$

where $z_{2}$ and $z_{3}$ are number of teeth of planet wheel and epicycle correspondingly.

One sliding surface of bearing is a part of carrier and another is a part of planet wheel. The relative motion of sliding surfaces with angular speed $\omega_{2}$ causes fluid velocity distribution and therefore hydrodynamic pressure in bearing gap. Planet wheel participating in compound motion: rotation with carrier $(H)$ with angular speed $\omega_{H}$ and rotation around planet wheel bearing axis with angular speed $\omega_{2}$. Thus both sliding surfaces " $H$ " and "2" (Figure $1 b$ ) of planet wheel journal bearing rotating with angular speed $\omega_{H}$ about axis $\mathrm{O}_{H}$. And sliding surface "2" also rotating about axis $\mathrm{O}_{2}$. Accelerations in fluid film are defined by sliding surfaces motion. Accelerations for sliding surfaces in bearing are 
shown in Figure $1 b$. Acceleration in fluid film are consist of vector sum of transfer $\left(a_{e}\right)$, relative $\left(a_{l u b}\right)$ and Coriolis $\left(a_{c}\right)$ acceleration components which are determined as follows in cylindrical coordinate system $\mathrm{O}_{2} r \varphi z$.

$$
\begin{gathered}
\bar{a}_{e}=-\omega_{H}^{2}\left(R+r_{H} \cos \varphi\right) \cdot \bar{e}_{r}+\omega_{H}^{2} r_{H} \sin \varphi \cdot \bar{e}_{\varphi} ; \\
\bar{a}_{l u b}=-\left(u^{2} / R\right) \cdot \bar{e}_{r} ; \\
\bar{a}_{c}=-2 \omega_{H} v \cdot \bar{e}_{\varphi}+2 \omega_{H} u \cdot \bar{e}_{r},
\end{gathered}
$$

where $R$ is a bearing radius, $r_{H}=O_{H} O_{2}$ is a planet wheel center radius, $u$ and $v$ are the fluid velocity components as they shown in Figure 2. In Figure 2: $h$ is a fluid film thickness. Acceleration components expressions (2) allows to write a unit inertia force in fluid film as follows

$$
\begin{gathered}
\bar{F}_{i}=-\bar{a}_{e}-\bar{a}_{c}-\bar{a}_{l u b}=\left(-\omega_{H}^{2} r_{H} \sin \varphi+2 \omega_{H} v\right) \cdot \bar{e}_{\varphi}+ \\
+\left[\omega_{H}^{2}\left(R+r_{H} \sin \varphi\right)-2 \omega_{H} u+\frac{u^{2}}{R}\right] \cdot \bar{e}_{r}+0 \cdot \bar{e}_{z}= \\
=f_{s} \cdot \bar{e}_{\varphi}+f_{y^{\prime}} \cdot \bar{e}_{r}+f_{z} \cdot \bar{e}_{z},
\end{gathered}
$$

where $f_{s}, f_{y}$ and $f_{z}$ are fluid inertia force components in coordinate system $\mathrm{O}_{2} r \varphi z$. Fluid inertia force in form (3) should be taken into account in mathematical model of fluid flow in bearing.
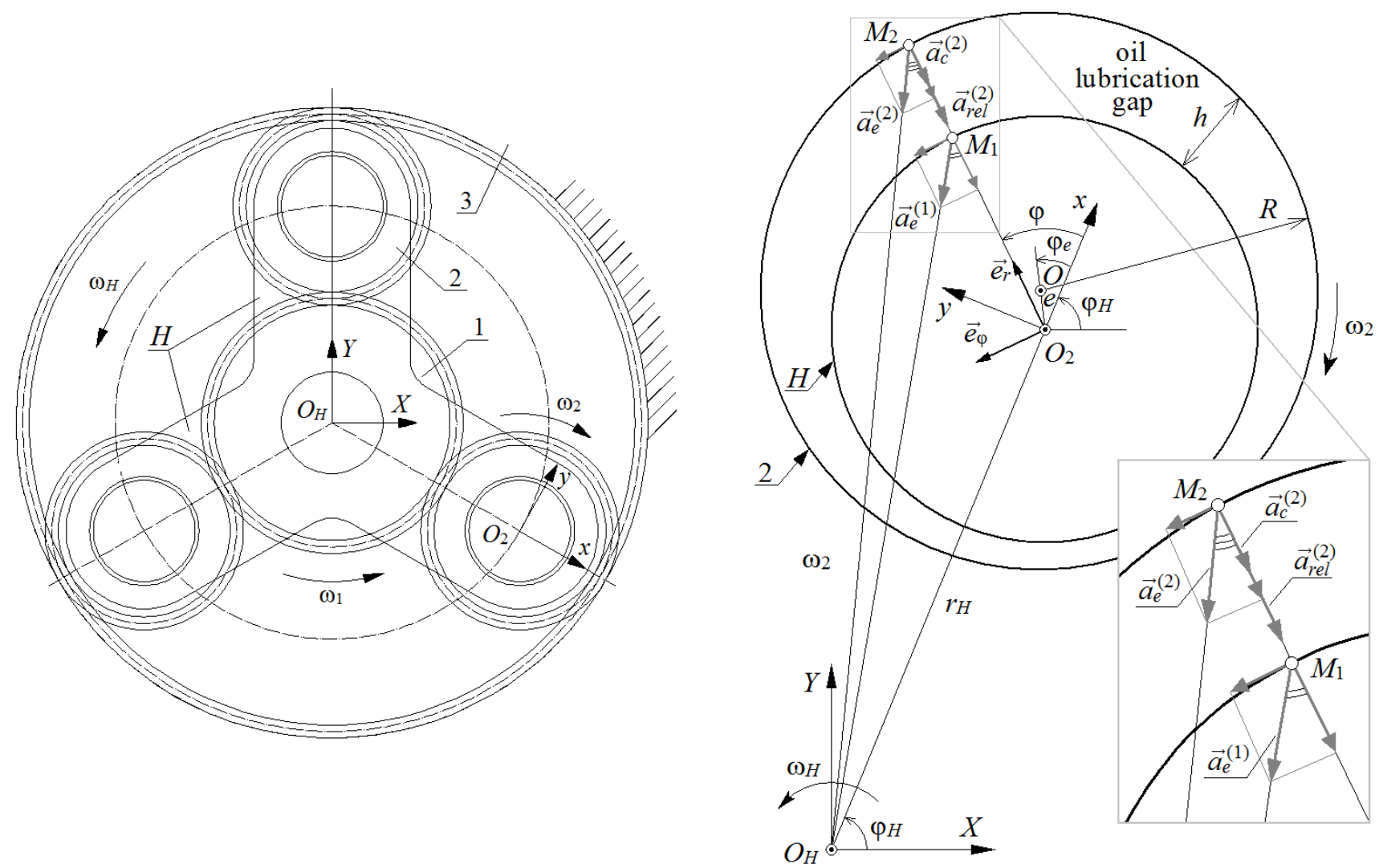

a)

b)

Fig. 1. Planetary gear; a) structure: 1 - sun wheel; $\mathrm{H}$ - carrier; 2 - planet wheel; 3 - ring wheel; b) accelerations on sliding surfaces of journal bearing

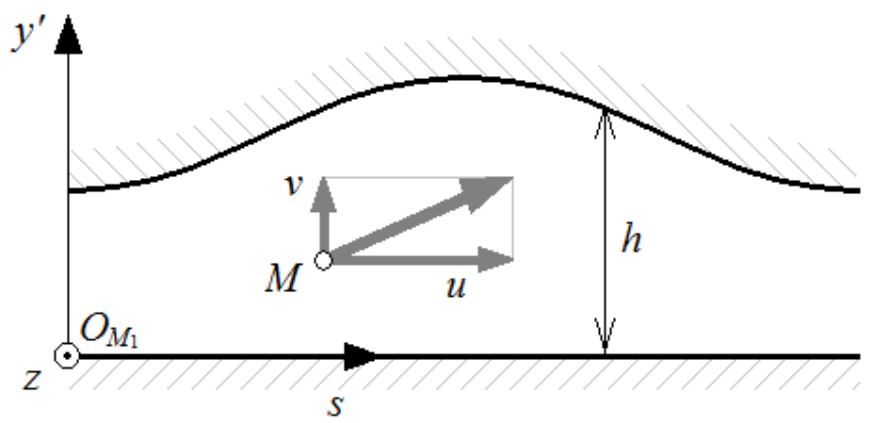

Fig. 2. Velocities in lubrication gap 


\section{MATHEMATICAL MODEL}

Required modification of Reynolds equation taking into account mean values of fluid inertia force (3) one can receive from Navier-Stokes and continuity equations [4] applying assumptions for fluid flow in thin film. These assumptions allow introducing dimensionless variables, coordinates and constants in following form [5]

$$
\begin{gathered}
s=\bar{s} R, y^{\prime}=y h, z=\bar{z} R, \\
u=\bar{u} V, v=\bar{v}(h / R) V, w=\bar{w} V,
\end{gathered}
$$

$$
\bar{h}=\frac{h}{h_{m}}, \bar{p}=\frac{p}{p_{0}}, \bar{t}=\frac{V t}{R}, \psi=\frac{h_{m}}{R}, \operatorname{Re}=\frac{\rho V h}{\eta},
$$

where $V=\omega_{2} R$ is a specific linear velocity on sliding surfaces, $h_{m}$ is an average fluid film thickness, $\rho$ and $\eta$ are fluid film density and dynamic viscosity correspondingly. Taking into account nondimensionalization (4) Navier-Stokes equations for laminar isothermal flow of incompressible fluid in bearing can be written in coordinate system sy'z (Figure 2) as follows

$$
\left\{\begin{array}{l}
\psi \operatorname{Re} \frac{d \bar{u}}{d \bar{t}}=\psi \operatorname{Re} \bar{f}_{s}-\psi \frac{p_{0} h_{m}}{\eta V} \frac{\partial \bar{p}}{\partial \bar{s}}+\frac{1}{\bar{h}^{2}} \frac{\partial^{2} \bar{u}}{\partial \bar{y}^{2}}+\psi^{2}\left(\frac{\partial^{2} \bar{u}}{\partial \bar{s}^{2}}+\frac{\partial^{2} \bar{u}}{\partial \bar{z}^{2}}\right)+\frac{\psi^{2}}{3} \frac{\partial}{\partial \bar{s}} \operatorname{div}\left(\bar{v}_{l u b}\right), \\
\psi \operatorname{Re} \bar{h} \frac{d \bar{v}}{d \bar{t}}=\psi \operatorname{Re} \bar{f}_{y^{\prime}}-\frac{p_{0} h_{m}}{\eta V} \frac{1}{\bar{h}} \frac{\partial \bar{p}}{\partial \bar{y}}+\psi^{3} \bar{h}\left(\frac{\partial^{2} \bar{v}}{\partial \bar{s}^{2}}+\frac{\partial^{2} \bar{v}}{\partial \bar{z}^{2}}\right)+\frac{\psi}{3} \frac{1}{\bar{h}} \frac{\partial}{\partial \bar{y}} \operatorname{div}\left(\bar{v}_{l u b}\right)+\psi \frac{1}{\bar{h}} \frac{\partial^{2} \bar{v}}{\partial \bar{y}^{2}}, \\
\psi \operatorname{Re} \frac{d \bar{w}}{d \bar{t}}=-\psi \frac{p_{0} h_{m}}{\eta V} \frac{\partial \bar{p}}{\partial \bar{z}}+\frac{1}{\bar{h}^{2}} \frac{\partial^{2} \bar{w}}{\partial \bar{y}^{2}}+\psi^{2}\left(\frac{\partial^{2} \bar{w}}{\partial \bar{s}^{2}}+\frac{\partial^{2} \bar{w}}{\partial \bar{z}^{2}}\right)+\frac{\psi^{2}}{3} \frac{\partial}{\partial \bar{z}} \operatorname{div}\left(\bar{v}_{l u b}\right),
\end{array}\right.
$$

where dimensionless fluid inertia forces

$$
\begin{gathered}
\bar{f}_{s}=-\frac{\omega_{H}^{2}}{\omega_{2}^{2}} \frac{r_{H}}{R} \sin \varphi+2 \psi \frac{\omega_{H}}{\omega_{2}} \bar{h} \overline{\mathrm{v}}, \\
\bar{f}_{y^{\prime}}=\frac{\omega_{H}^{2}}{\omega_{2}^{2}}\left(1+\frac{r_{H}}{R} \cos \varphi\right)-2 \frac{\omega_{H}}{\omega_{2}} \bar{u}+\bar{u}^{2}
\end{gathered}
$$

All dimensionless variables and their derivatives have same order of magnitude. Thus order of magnitude of terms in (5) and (6) are specified by coefficient $\psi \sim 10^{-3}$ and Reynolds number $R e$. While neglecting of terms with less order of magnitude in (5) and (6) the Navier-Stokes equations will have form

$$
\frac{\partial}{\partial s}\left(h^{3} \frac{\partial p}{\partial s}\right)+\frac{\partial}{\partial z}\left(h^{3} \frac{\partial p}{\partial z}\right)=-6 \omega_{2} \eta R \frac{\partial h}{\partial s}-\rho \omega_{H}^{2} r_{H} \frac{\partial}{\partial s}\left(h^{3} \sin \frac{s}{R}\right) .
$$

Numerical solution of Reynolds equation (8) carried out using finite-element discretization procedure. Special triangular finite element for fluid film pressure is used. Developed bearing model taking into account carrier and planet wheel rotations and allows calculating pressure distribution in bearing for various relations between $\omega_{H}$ and $\omega_{2}$ for any shaft journal eccentricity caused by gears of planet wheel with sun wheel and epicycle.

\section{MODEL VERIFICATION AND RESULTS}

Bearing mathematical model was verified for the case of long bearing by comparison with analytical solution and numerical solution in STAR-CD software based on full Navier-Stokes equations. Calculations was performed for parameters: bearing radius $R=15 \mathrm{~mm}$, distance between

$$
\left\{\begin{array}{l}
\rho \frac{d u}{d t}=-\rho \omega_{H}^{2} r_{H} \sin \varphi-\frac{\partial p}{\partial s}+\eta \frac{\partial^{2} u}{\partial y^{\prime 2}} \\
0=-\frac{\partial p}{\partial y^{\prime}} \\
\rho \frac{d w}{d t}=-\frac{\partial p}{\partial z}+\eta \frac{\partial^{2} w}{\partial y^{\prime 2}}
\end{array},\right.
$$

and modification of Reynolds equation with taking into account centrifugal inertia forces for planet wheel bearing will receive from (7) as follows planet wheel and carrier centers $r_{H}=167 \mathrm{~mm}$, radial gap in bearing $\delta=0,05 \mathrm{~mm}$, oil viscosity $\eta=0,02 \mathrm{~Pa} \cdot \mathrm{s}$ and density $\rho=900 \mathrm{~kg} / \mathrm{m}^{3}$, planet wheel rotation frequency $n_{2}=15000 \mathrm{rpm}$, carrier rotation frequency $n_{H}=6000 \mathrm{rpm}$, ambient pressure $p_{0}=0,1 \mathrm{MPa}$. A model of long bearing was created in STAR-CD for verification (Figure 3). Simulation of long bearing was performed by special boundary conditions on bearing calculation model faces: Velocity and acceleration components in direction $\mathrm{Oz}$ is equal to zero. STAR-CD model is used a full Navier-Stokes equation for laminar flow at constant temperature distribution without slipping motion of fluid. Finite volumes method is used for calculation. Bearing model in STAR-CD take into account all components of fluid inertia force (3). Centrifugal inertia forces regarding to planet wheel rotation around carrier center are simulated by 
rotation of coordinate system with center in $\mathrm{O}_{2}$ around coordinate system with center in $\mathrm{OH}_{\mathrm{H}}$

Verification results presented in Figure $4 a$ for three values of eccentricity ratio $\mathrm{x}: 0.2,0.4$ and 0.6 . Comparison of results shows good correspondence of two models. In Figure $4 \mathrm{c}$ the pressure distribution for two models for conditions of rotating carrier and nonrotating planet wheel ( $n_{2}=0 \mathrm{rpm}, n_{H}=6000 \mathrm{rpm}$ ) shows that centrifugal fluid inertia forces producing positive relative pressure in zone the most distant from carrier center and negative relative pressure in zone most close to carrier center.

Oil pressure in bearing presented in Figures 4, 5 and 6 are absolute due to boundary conditions for ambient pressure taken into account. Both Reynolds and STAR-CD mathematical models obtaining in results negative absolute pressure values. Negative value of oil pressure is shown by dash line only in Figure 4 for better understanding of comparison between Reynolds and STAR-CD models. In zone where absolute pressure less then ambient pressure the cavitation effects take place and absolute pressure in this zone is not less than zero. Current models (Reynolds and STAR-CD) don't take into account discontinuous flow of fluid. However pressure in unpressurized zone gives small income to bearing carrying force and can be neglected. Thus in verificated model of journal bearing based on Reynolds equation (Figure 5 ) all pressures less than zero are set equal to zero.

Pressure distribution in bearing for listed above parameters for bearing with length to diameter ratio $(I / d)$ equal to 1 is calculated for different shaft journal eccentricity in bearing. In Figures 5 and 6 pressure distribution for different directions of constant shaft journal eccentricity (eccentricity ratio $x=0,3$ ) is shown. Centrifugal forces have divergent influence on total planet bearing pressure depending upon direction of bearing eccentricity related to line connected carrier and planet wheel centers and corresponding to eccentricity hydrodynamic pressure. Thus for hydrodynamic pressure concentrated in fluid film most distant from carrier center (for example: angles $\pi / 2$ and $\pi$ in Figures 5 and 6) centrifugal forces increasing total oil pressure in bearing. For hydrodynamic pressure concentrated in fluid film most close to carrier center (for example: angles 0 and $3 \pi / 2$ in Figure 5) total oil pressure decreasing. This also leads for decreasing of positive pressure zone for some eccentricity directions. In Figures 5 and 6: for angle 0 pressure zone increasing, and for angle $3 \pi / 2$ - decreasing.

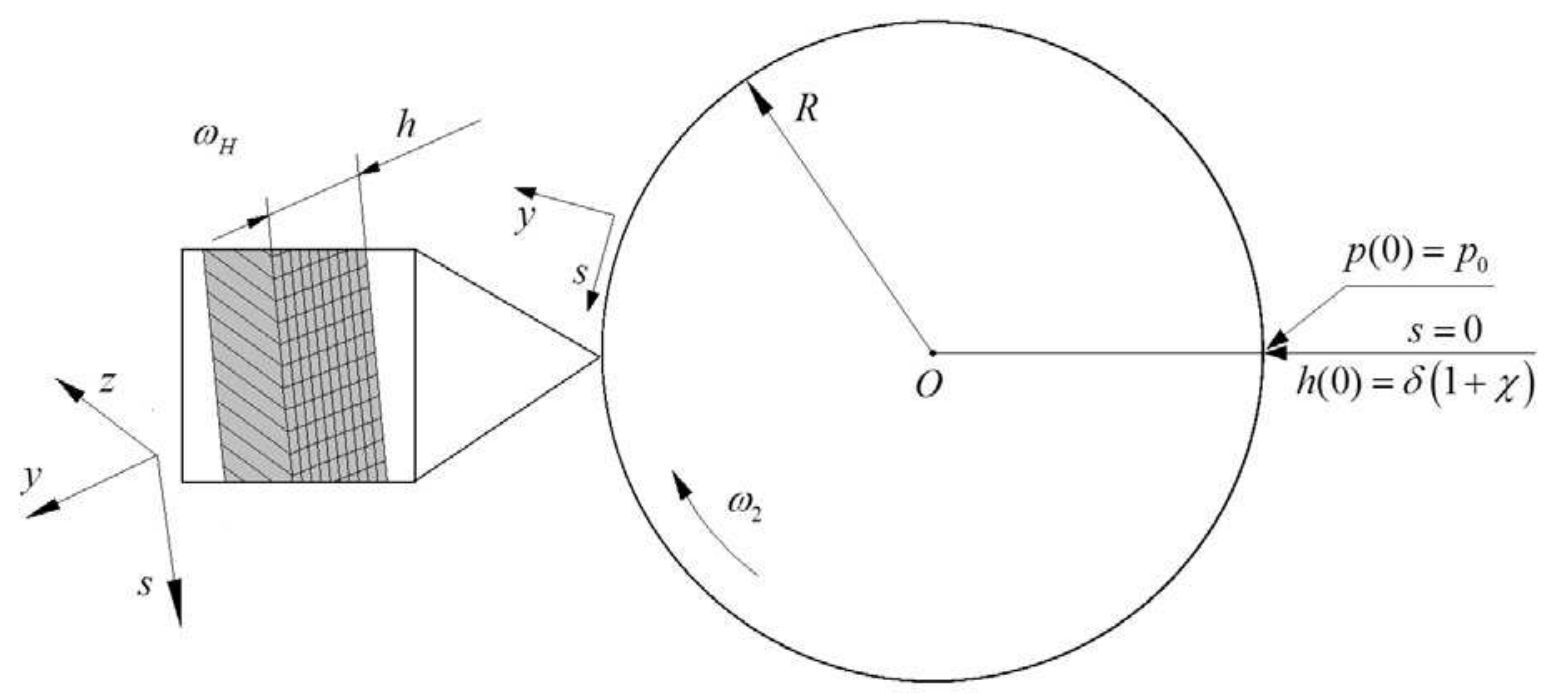

Fig. 3. STAR-CD model 
$p, \mathrm{MPa}$
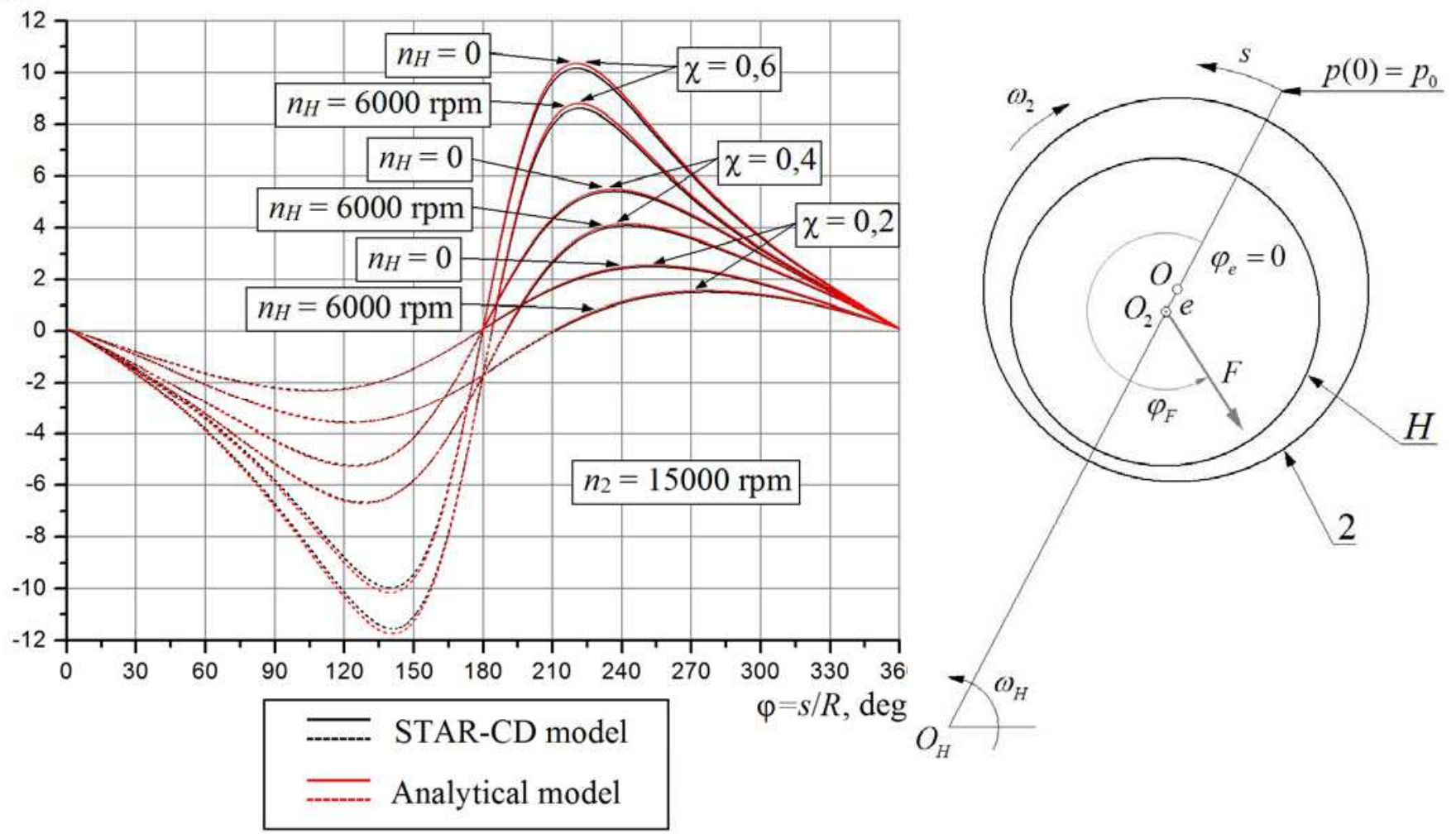

a)

b)

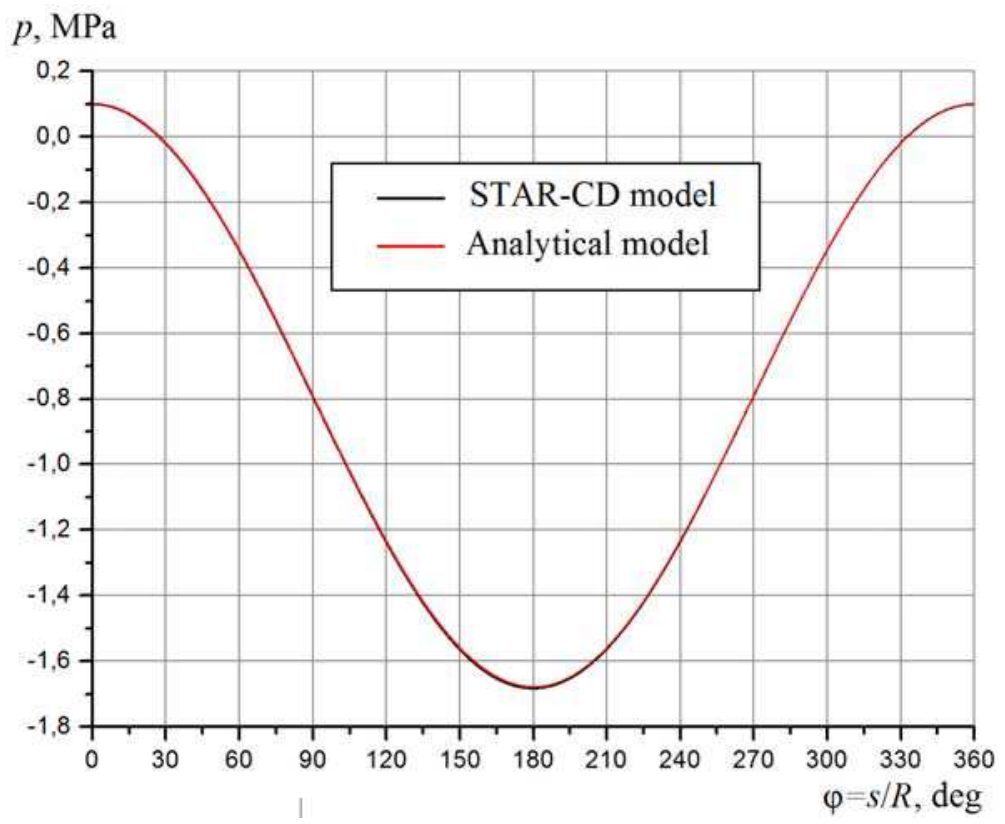

c)

Fig. 4. Pressure distribution in bearing: a) comparison of STAR-CD and Reynolds models for rotating and fixed carrier; b) scheme of shaft journal displacements in bearing; c) contribution of pressure caused by centrifugal forces 

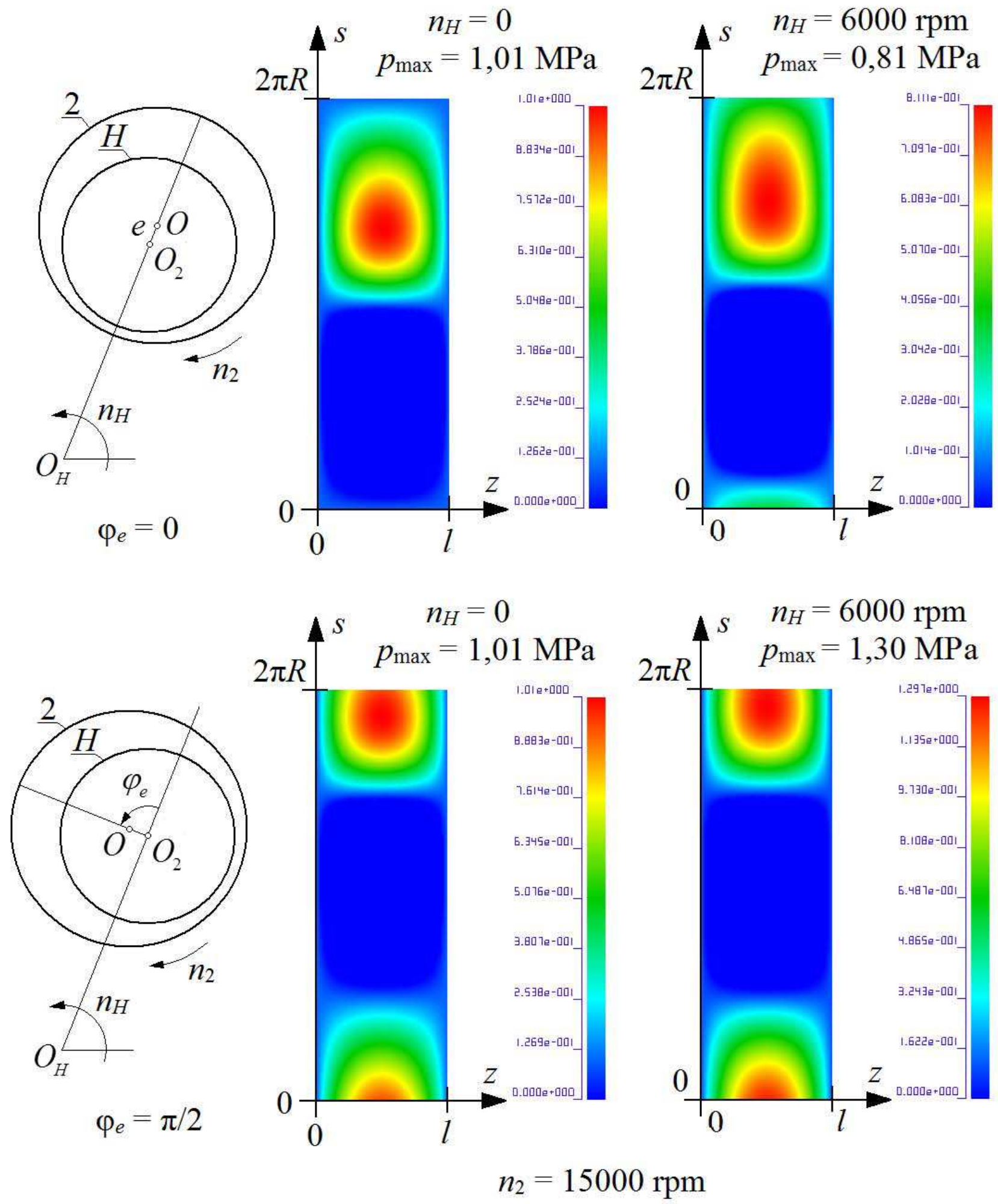

Fig. 5. Pressure distribution on bearing for different eccentricity directions 


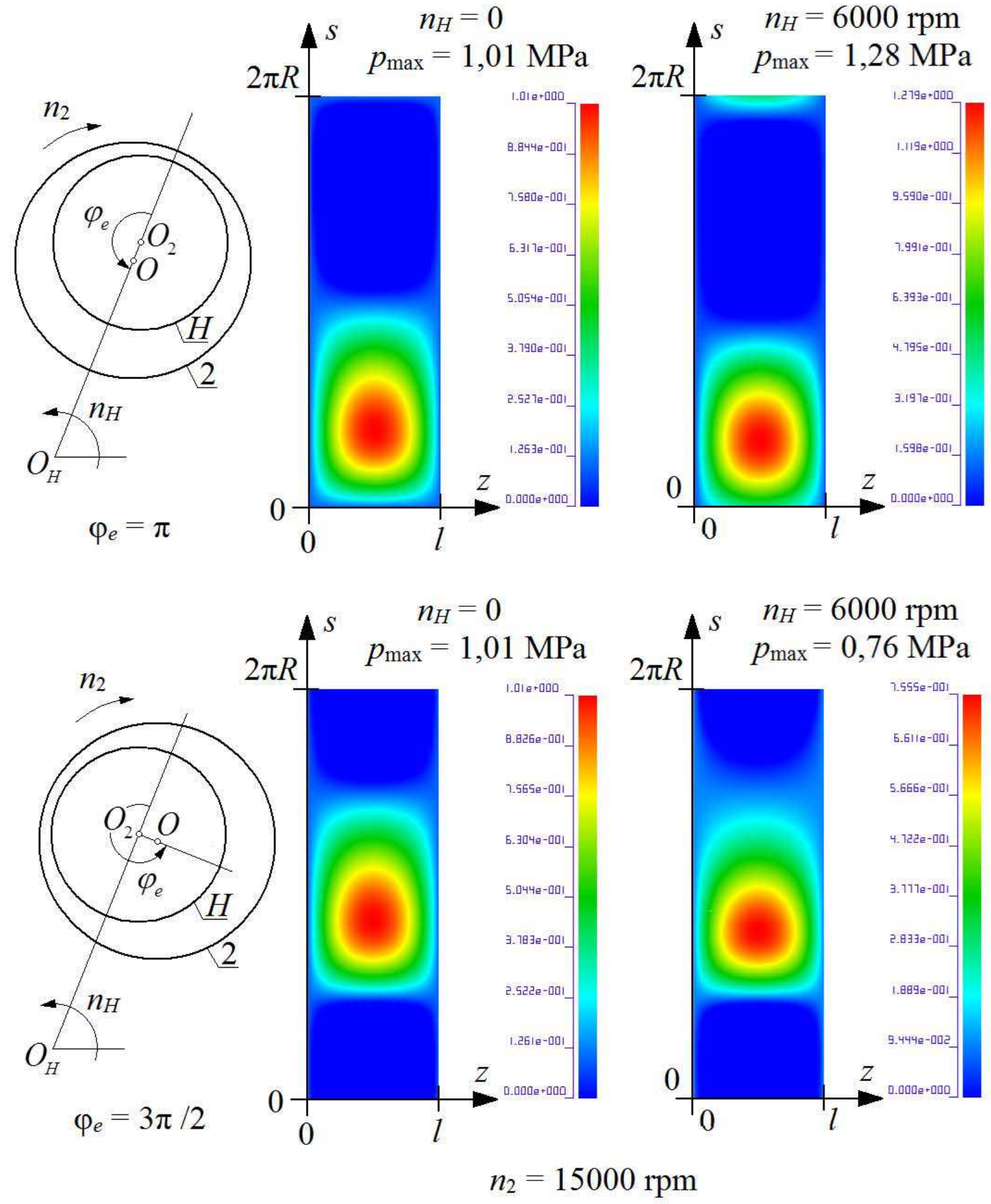

Fig. 6. Pressure distribution on bearing for different eccentricity directions 


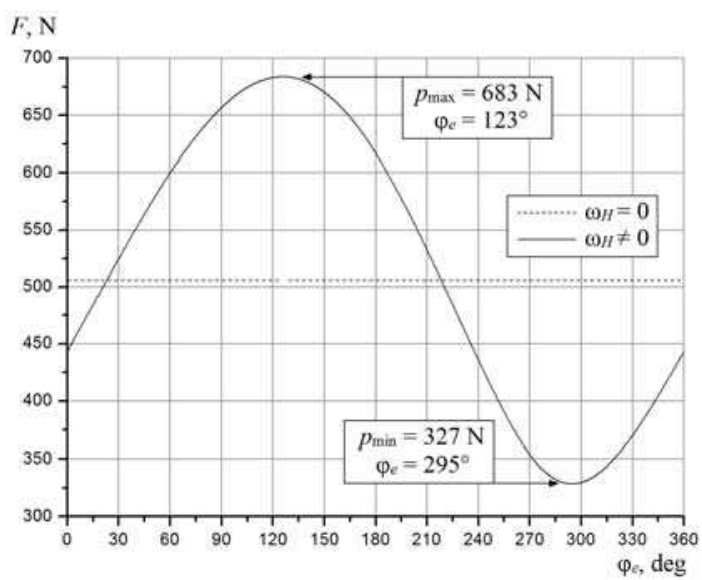

a) $X=0.2$

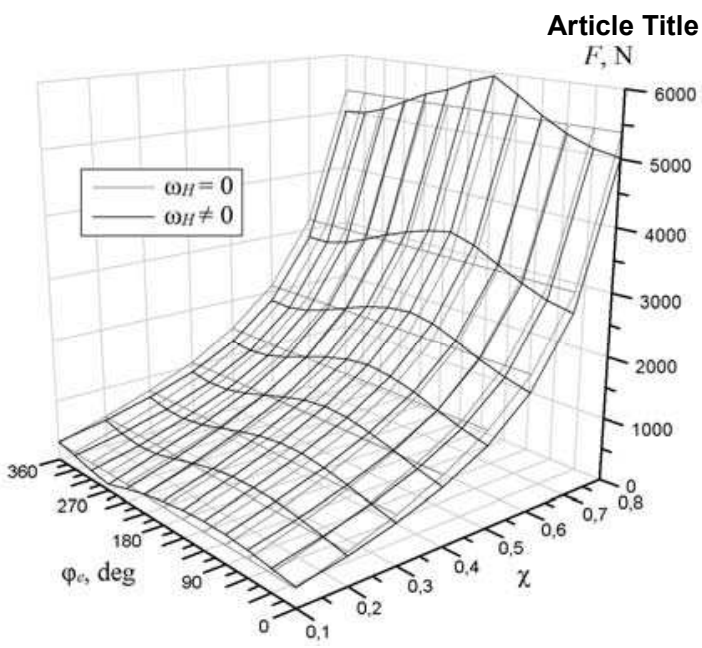

b)

Fig. 7. Bearing carrying force for different eccentricity directions

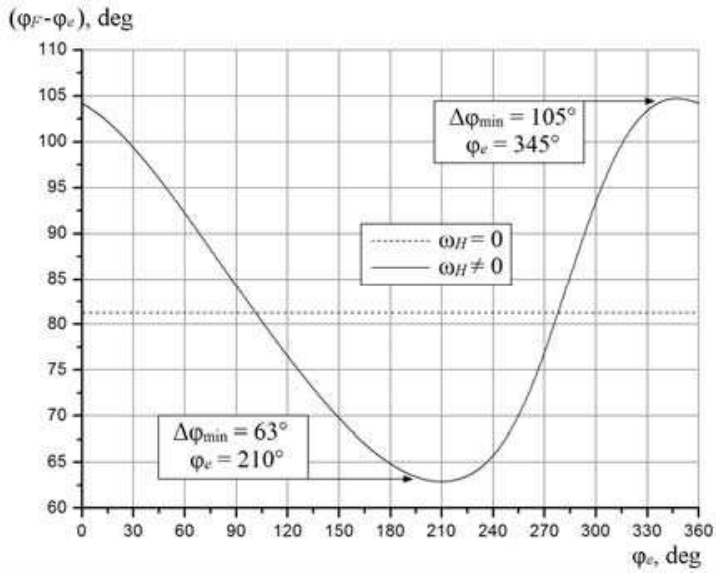

a) $X=0.2$

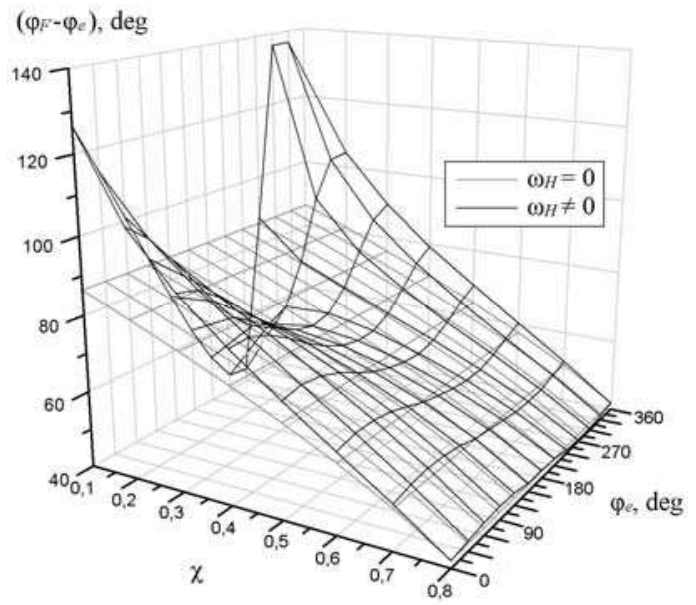

b)

Fig. 8. Attitude angle for different eccentricity directions

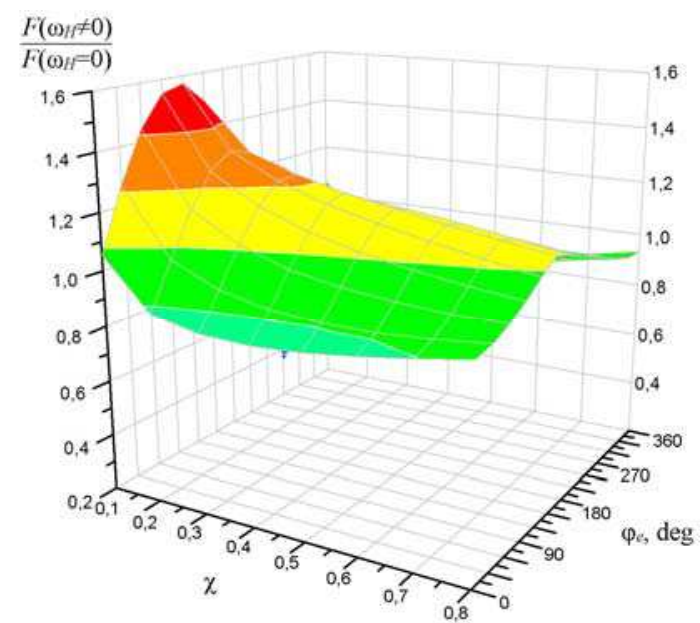

a)

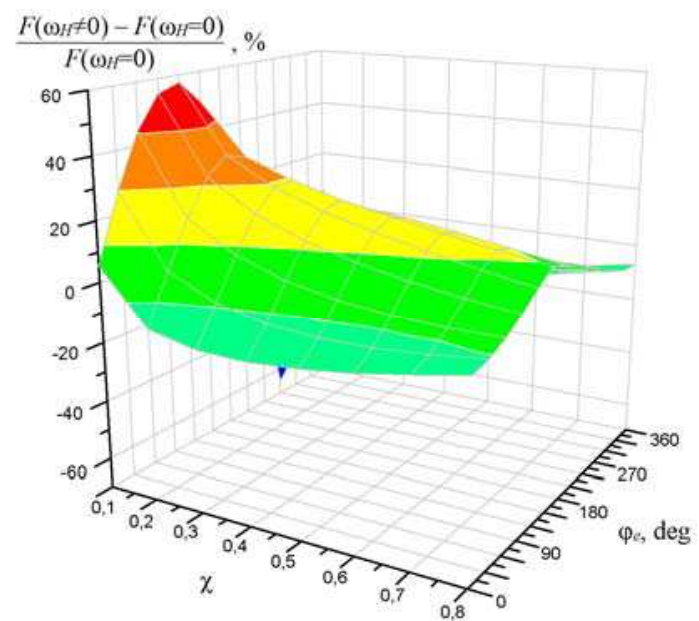

b)

Fig. 9. Bearing carrying force ratio for carrying force in bearing with fixed axis

Results for pressure distribution for constant eccentricity ratio and various eccentricity directions provide variable bearing carrying force (Figure 7 ) and attitude angle (Figure 8). Under the bearing carrying force $F$ the integral force of oil pressure in bearing which is equilibrating rotor reaction in bearing is represented. For full $360^{\circ}$ bearing with smooth sliding surfaces and fixed axis the carrying force is constant for constant eccentricity. However centrifugal inertia forces action leads to cyclic change of carrying force with maximum value at angle $\varphi_{\mathrm{e}}=123^{\circ}$ and minimum value at angle $\varphi_{e}=295^{\circ}$. Maximal force value greater then carrying force in bearing with fixed axis for $35.2 \%$ and minimum value 
is less for $35.4 \%$. There are two angles $\varphi_{e}=23^{\circ}$ and $\varphi_{e}=218^{\circ}$ for which centrifugal inertia forces have a negligible influence.

Centrifugal inertia forces provide a fixed contribution in bearing pressure for constant rotation frequencies. Centrifugal inertia forces will have a smaller influence on total pressure in case of eccentricity value growing. This is explained by growing of hydrodynamic pressure for growing eccentricity while centrifugal inertia pressure leaves constant. It is demonstrated on graph in Figure 9 where bearing carrying forces ratio (Figure 9a) and relative changing in percent (Figure 9b) for two cases of fixed bearing axis and planet wheel bearing are shown.

\section{CONCLUSION}

Developed mathematical model based on modified Reynolds equation taking into account centrifugal and Coriolis forces acting in fluid in bearing gap. This model gives ability for more accurate determination of planet wheel position in bearing and calculation of bearing carrying force depending upon load comes from gear.

The sufficient influence of centrifugal inertia forces on pressure distribution in bearing and correspondingly on carrying force and attitude angle is shown. Changing of carrying force due to action of centrifugal forces in range of eccentricity ratio values $X=0.2 . .0 .6$ are in range from $35 \%$ for eccentricity ratio 0.2 to $16 \%$ for eccentricity ratio 0.6 (in percent of carrying force for fixed axis bearing, Figure 9b).

\section{REFERENCES}

[1] Kurzke J. Fundamental Differences Between Conventional and Geared Turbofans. Proceedings of ASME Turbo Expo 2009: Power for Land, Sea and Air, Orlando, Florida, USA, June 8-12, 2009.

[2] Riegler C. and Bichlmaier C. The Geared Turbofan Technology Opportunities, Challenges, and Readiness Status. Proceedings of the $1^{\text {st }}$ CEAS European Air and Space Conference, Berlin, Germany, September 2007.

[3] Braun M. J. and Hannon W. M. Cavitation Formation and Modeling for Fluid Film Bearings: A Review. Proceedings of the Institution of mechanical Engineers, Part J: Journal of Engineering Tribology, 2010, Vol. 224, 9, p. 839-863.

[4] Temis J.M., Temis M.J. Stiffness and Damping Characteristics of Journal Bearing with Flexible Working Surfaces // J. of Friction and Wear. 2007 (28) No 2, P. 128137. (In Russ.)

[5] Temis J.M. and Temis M.J. Contribution of Bearing Structure in Gas Turbine Power Unit Rotor Dynamics. Proc. 3rd Int. Symp. on Stability Control of Rotating Machinery (ISCORMA-3), Cleveland, USA, 2005, p. 570-581.

${ }^{[6]}$ Constantinescu V. N. Gas lubrication. Mashinostroenie, 1968, 712 p. (in Russian) 